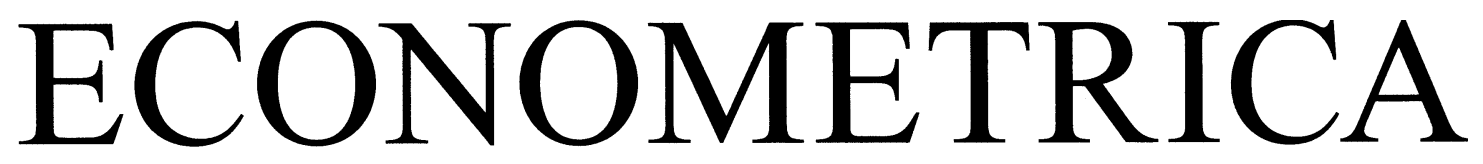

JOURNAL OF THE ECONOMETRIC SOCIETY

An International Society for the Advancement of Economic Theory in its Relation to Statistics and Mathematics

http://www.econometricsociety.org/

Econometrica, Vol. 81, No. 6 (November, 2013), 2535-2559

ON THE TESTABILITY OF IDENTIFICATION IN SOME NONPARAMETRIC MODELS WITH ENDOGENEITY

IVAN A. CANAY

Northwestern University, Evanston, IL 60208, U.S.A.

ANDRES SANTOS

University of California-San Diego, La Jolla, CA 92093, U.S.A.

AZEEM M. SHAIKH

University of Chicago, Chicago, IL 60657, U.S.A.

The copyright to this Article is held by the Econometric Society. It may be downloaded, printed and reproduced only for educational or research purposes, including use in course packs. No downloading or copying may be done for any commercial purpose without the explicit permission of the Econometric Society. For such commercial purposes contact the Office of the Econometric Society (contact information may be found at the website http://www.econometricsociety.org or in the back cover of Econometrica). This statement must be included on all copies of this Article that are made available electronically or in any other format. 


\title{
NOTES AND COMMENTS \\ ON THE TESTABILITY OF IDENTIFICATION IN SOME NONPARAMETRIC MODELS WITH ENDOGENEITY
}

\begin{abstract}
By Ivan A. CANay, Andres SAntos, And AZEEM M. ShaiKH ${ }^{1}$
This paper examines three distinct hypothesis testing problems that arise in the context of identification of some nonparametric models with endogeneity. The first hypothesis testing problem we study concerns testing necessary conditions for identification in some nonparametric models with endogeneity involving mean independence restrictions. These conditions are typically referred to as completeness conditions. The second and third hypothesis testing problems we examine concern testing for identification directly in some nonparametric models with endogeneity involving quantile independence restrictions. For each of these hypothesis testing problems, we provide conditions under which any test will have power no greater than size against any alternative. In this sense, we conclude that no nontrivial tests for these hypothesis testing problems exist.
\end{abstract}

KEYWORDS: Instrumental variables, identification, completeness, bounded completeness.

\section{INTRODUCTION}

INSTRUMENTAL VARIABLES (IV) METHODS have a prominent role in econometrics due to their ability to uncover causal effects in observational studies. Though traditionally parametric in nature, an important literature has extended IV methods to a variety of nonparametric settings. Among these extensions, of particular prominence is the additively separable specification in which, for an outcome of interest $Y$, a regressor $W$, and an instrument $Z$, it is assumed that

$$
Y=\theta(W)+\varepsilon,
$$

with $\varepsilon$ mean independent of $Z$. Under the maintained assumption that the model is correct, Newey and Powell (2003) showed identification of $\theta$ to be equivalent to the joint distribution of $W$ and $Z$ satisfying a completeness condition. Complementing their prevalent use in statistics (Lehmann and Scheffé $(1950,1955))$, completeness conditions have since then been widely used in econometrics - see Hall and Horowitz (2005), Blundell, Chen, and Kristensen

\footnotetext{
${ }^{1}$ We thank James Stock, Victor Chernozhukov, and four anonymous referees whose valuable suggestions helped greatly improve the paper. We are also indebted to Xiaohong Chen, Joel Horowitz, Patrick Kline, Whitney Newey, Elie Tamer, Daniel Wilhelm, and numerous seminar participants for valuable comments. The research of the first author has been supported by National Science Foundation Grant SES-1123586. The research of the third author has been supported by National Science Foundation Grants DMS-0820310 and SES-1227091, and the Alfred P. Sloan Foundation.
} 
(2007), Hu and Schennach (2008), Cunha, Heckman, and Schennach (2010), Berry and Haile (2010a), Darolles, Fan, Florens, and Renault (2011), and the references therein.

Despite the widespread use of completeness conditions in econometrics, little evidence has been provided about their reasonableness in applications of interest to economists. We note, however, that since completeness conditions impose restrictions on the distribution of the observed data, it is potentially possible to provide such evidence by testing the validity of these assumptions. This paper explores precisely this possibility. Specifically, we study whether it is possible to test the null hypothesis that a completeness condition does not hold against the alternative that it does hold. Such a hypothesis testing problem is consistent with a setting in which a researcher wishes to assert that the model is identified and hopes to find evidence in favor of this claim in the data. This setup is also analogous to tests of rank conditions in linear models with endogeneity, where the null hypothesis is that of rank-deficiency.

In this paper, we show that, under commonly imposed restrictions on the distribution of the data, the null hypothesis that the completeness condition does not hold is in fact untestable. Formally, we establish that any test will have power no greater than size against any alternative. It is therefore not possible to provide empirical evidence in favor of the completeness condition by means of such a test. This conclusion is in contrast to the testability of a failure of the rank condition in linear specifications of $\theta$, for which nontrivial tests do exist under reasonable assumptions. Thus, while completeness conditions provide an intuitive generalization of the rank condition in a linear specification, the empirical implications of these assumptions are substantially different in this sense.

We additionally derive analogous results in two other prominent nonparametric models with endogeneity. The first such model follows the specification in (1) with a prespecified conditional quantile of $\varepsilon$ assumed independent of $Z$ - see Chernozhukov and Hansen (2005), Horowitz and Lee (2007), Gagliardini and Scaillet (2012), and Chen and Pouzo (2012). The second such model follows a specification in which $\theta$ is allowed to depend nonseparably on both $W$ and $\varepsilon$, with the dependence on $\varepsilon$ being monotonic, and all conditional quantiles of $\varepsilon$ assumed independent of $Z$-see Chernozhukov and Hansen (2005), Imbens and Newey (2009), Torgovitsky (2012), d'Haultfoeuille and Février (2012), and Berry and Haile (2009, 2010b). Due to the nonlinear nature of such models, simple, global rank conditions such as completeness conditions are unavailable. For this reason, we instead directly consider the testability of the null hypothesis that identification fails against the alternative hypothesis that it holds. Analogously to our results concerning the testability of completeness conditions, we obtain conditions under which no nontrivial tests exist for these hypothesis testing problems either.

We emphasize that our results should not be interpreted as an indictment against nonparametric methods in models with endogeneity. The nontestability of completeness conditions or identification does not imply that these 
assumptions are false. The impossibility of providing supporting empirical evidence by means of such a test, however, does suggest that it is prudent to justify their use with alternative arguments in favor of their validity. Indeed, much research relies on assumptions that are untestable, but are nonetheless deemed reasonable on different grounds. Important progress in this regard has been recently made by Andrews (2011) and Chen, Chernozhukov, Lee, and Newey (2012), who established different genericity results for distributions satisfying completeness restrictions. Each of these results provides a sense under which completeness conditions are satisfied by "most" distributions in a large class of distributions. Thus, while completeness conditions may be untestable, one may argue that they are "commonly satisfied" by appealing to such results.

We further stress that completeness or identification assumptions are not necessary for conducting nonparametric inference in models with endogeneity. As is well understood in semiparametric models, certain inferential methods can be robust to whether identification is "strong," "weak," or partial-see, among others, Anderson and Rubin (1949), Kleibergen (2002), and Moreira (2003) for results in the classical, linear IV setting, and Chernozhukov, Hansen, and Jansson $(2007,2009)$ for extensions to settings with quantile restrictions. Insights from these papers have also been employed in the development of inferential procedures for general partially identified models-see Chernozhukov, Hong, and Tamer (2007) and Romano and Shaikh (2008). Our impossibility results emphasize the value of extending these approaches to nonparametric settings. Recent work toward this end includes Freyberger and Horowitz (2012) and Santos (2012), who, in the context of the first model we consider, developed inferential procedures that are robust to the possibility that the completeness condition fails and the model is therefore only partially identified. See also Chen, Tamer, and Torgovitsky (2011) for inferential procedures that are robust to partial identification in semiparametric likelihood models.

Finally, it is important to note that our results do not preclude the testability of completeness conditions or identification under alternative restrictions on $\theta$ or the distribution of the observed data. For instance, our arguments may not extend easily to settings where $\theta$ is restricted to be a density (Hoderlein, Nesheim, and Simoni (2012)) or is semiparametrically specified (Ai and Chen (2003)). We further discuss the possible implications of alternative restrictions on $\theta$ or the distribution of the observed data in the text-see Remarks 3.1-3.2 and the discussion following those remarks.

This paper contributes to an important literature on impossibility results in econometrics-see Leeb and Pötscher (2008) and Müller (2008) for recent examples, and Dufour (2003) for an excellent overview. First among such results is Bahadur and Savage (1956), who documented the impossibility of conducting nontrivial inference on the mean without appropriate restrictions on the distribution of the observed data. Romano (2004) later showed that this result is the consequence of the set of distributions satisfying the alternative hypothesis lying in the closure, under the Total Variation distance, of the set of 
distributions satisfying the null hypothesis. Intuitively, if, for every distribution satisfying the alternative hypothesis, there exists an arbitrarily "close" distribution satisfying the null hypothesis, then it will be impossible to discriminate between them from data-see Pötscher (2002) for related ideas. Our results share this intuition, but require novel arguments for showing that distributions for which a completeness condition or identification fails can approximate distributions for which it holds arbitrarily well in the Total Variation distance.

The remainder of the paper is organized as follows. Section 2 elaborates on the nature of our impossibility results, and reviews a general framework for deriving them. The main results are developed in Section 3. Section 4 briefly concludes. All proofs are contained in the Appendix.

\section{SETUP}

Before formally stating the three distinct hypothesis problems we consider, it will be useful to introduce some notation and elaborate on the nature of our impossibility results. Toward this end, we let $\left\{V_{i}\right\}_{i=1}^{n}$ be an independent and identically distributed (i.i.d.) sequence of random variables with distribution $P \in \mathbf{P}$, and denote by $P^{n}$ the $n$-fold product $\bigotimes_{i=1}^{n} P$. The hypothesis testing problems we study may then be expressed as

$$
H_{0}: P \in \mathbf{P}_{0} \quad \text { versus } H_{1}: P \in \mathbf{P}_{1},
$$

where $\mathbf{P}_{0}$ is the subset of $\mathbf{P}$ for which the null hypothesis holds and $\mathbf{P}_{1}=\mathbf{P} \backslash \mathbf{P}_{0}$ is the subset of $\mathbf{P}$ for which the alternative hypothesis holds. For a sequence of (possibly randomized) tests $\left\{\phi_{n}\right\}_{n=1}^{\infty}$, the corresponding size at sample size $n$ is given by

$$
\sup _{P \in \mathbf{P}_{0}} E_{P^{n}}\left[\phi_{n}\right] .
$$

In our analysis, we will show that under commonly imposed restrictions on the set of distributions $\mathbf{P}$, the three hypothesis testing problems we examine share the property that

$$
\sup _{P \in \mathbf{P}_{1}} E_{P n}\left[\phi_{n}\right] \leq \sup _{P \in \mathbf{P}_{0}} E_{P n}\left[\phi_{n}\right]
$$

for any sequence of (possibly randomized) tests $\left\{\phi_{n}\right\}_{n=1}^{\infty}$ and any sample size $n$. Equivalently, result (3) establishes that, for all tests, the power against any alternative $P \in \mathbf{P}_{1}$ is always bounded above by the size of the test. It also follows from such an assertion that any sequence of (possibly randomized) tests $\left\{\phi_{n}\right\}_{n=1}^{\infty}$ that controls asymptotic size at level $\alpha \in(0,1)$ will have asymptotic power no larger than $\alpha$ against any alternative. Formally, (3) immediately yields that

$$
\limsup _{n \rightarrow \infty} \sup _{P \in \mathbf{P}_{0}} E_{P^{n}}\left[\phi_{n}\right] \leq \alpha \quad \Longrightarrow \quad \limsup \sup _{n \rightarrow \infty} E_{P \in \mathbf{P}_{1}}\left[\phi_{n}\right] \leq \alpha .
$$


We therefore conclude that no nontrivial tests exist for hypothesis testing problems satisfying property (3). In other words, in such settings, no test can outperform a procedure that simply ignores the data and rejects the null hypothesis with probability $\alpha$.

\subsection{A Useful Lemma}

Underlying our arguments is a powerful result originally found in Romano (2004), which we restate due to its importance in our derivations. In the statement of the lemma, $\|P-Q\|_{\mathrm{TV}}$ denotes the Total Variation distance between probability measures $P$ and $Q$; for example, for $\lambda \equiv(P+Q) / 2$,

$$
\|P-Q\|_{\mathrm{TV}} \equiv \frac{1}{2} \int\left|\frac{d Q}{d \lambda}-\frac{d P}{d \lambda}\right| d \lambda .
$$

LEMMA 2.1: Let $\mathbf{M}$ denote the space of Borel probability measures on a separable metric space $\mathbf{V}$. Suppose $\mathbf{P} \subseteq \mathbf{M}$ and that $\mathbf{P}=\mathbf{P}_{0} \cup \mathbf{P}_{1}$. If, for each $P \in \mathbf{P}_{1}$, there exists a sequence $\left\{P_{k}\right\}_{k=1}^{\infty}$ in $\mathbf{P}_{0}$ with $\left\|P-P_{k}\right\|_{\mathrm{TV}} \rightarrow 0$ as $k \rightarrow \infty$, then every sequence of test functions $\left\{\phi_{n}\right\}_{n=1}^{\infty}$ satisfies

$$
\sup _{P \in \mathbf{P}_{1}} E_{P^{n}}\left[\phi_{n}\right] \leq \sup _{P \in \mathbf{P}_{0}} E_{P^{n}}\left[\phi_{n}\right] \quad \text { for all } n \geq 1 \text {. }
$$

Heuristically, Lemma 2.1 states that if each $P \in \mathbf{P}_{1}$ is on the boundary of the set of distributions satisfying the null hypothesis, then, by continuity, the probability of rejection under any $P \in \mathbf{P}_{1}$ must be no larger than the size of the test. Theorem 1 in Romano (2004) establishes that the appropriate topology for this purpose is that induced by the Total Variation distance. A metric compatible with weak convergence, such as the Lévy-Prokhorov metric, would be insufficient, as it would not guarantee convergence of integrals defining rejection probabilities. By contrast, the Total Variation distance between two measures $P$ and $Q$ is intimately related to the statistical properties of the best test for distinguishing between $P$ and $Q$ (Le Cam (1986)). For this reason, some authors have referred to the Total Variation distance as the "testing metric" (Donoho (1988)).

In each of the three hypothesis testing problems we consider, we establish nonexistence of nontrivial tests by constructing, for each $P \in \mathbf{P}_{1}$, a sequence $\left\{P_{k}\right\}_{k=1}^{\infty}$ in $\mathbf{P}_{0}$ with $\left\|P-P_{k}\right\|_{\mathrm{TV}} \rightarrow 0$ as $k \rightarrow \infty$ and applying Lemma 2.1. It is worth emphasizing that the Total Variation metric plays no role in determining $\mathbf{P}$ nor the null and alternative hypotheses. Indeed, $\mathbf{P}$ may possess other natural topologies, such as that induced by the Euclidean metric in parametric models, or that induced by a Hölder norm in sets of smooth densities. However, Lemma 2.1 reveals that, regardless of what topology $\mathbf{P}$ originally has, for our purposes we must examine $\mathbf{P}$ under the topology induced by the Total Variation metric. 
It would be natural to interpret Lemma 2.1 as a negative result, but we believe it is also instructive to emphasize its constructive nature. In particular, the converse of Lemma 2.1 implies that a necessary condition for the existence of nontrivial tests is that there is a $P \in \mathbf{P}_{1}$ that is not in the closure of $\mathbf{P}_{0}$ with respect to $\|\cdot\|_{\mathrm{TV}}$. Thus, an often fruitful approach for restoring testability has been to restrict $\mathbf{P}$ in a manner that ensures that this is the case. For example, Romano (2004) showed that the original impossibility result of Bahadur and Savage (1956) can be circumvented by restricting $\mathbf{P}$ to be a set of distributions satisfying a uniform integrability condition. This restriction on $\mathbf{P}$, and closely related ones, have been used widely in the recent literature on inference in partially identified models-see, for example, Romano and Shaikh (2008), Andrews and Guggenberger (2009), and Andrews and Soares (2010). Similarly, uniformly valid confidence regions for infinite-dimensional parameters have been obtained by restricting $\mathbf{P}$ through smoothness assumptions (Robins and van der Vaart (2006), Giné and Nickl (2010)) or shape restrictions (Hengartner and Stark (1995)). See also Romano and Shaikh (2012) for restrictions on $\mathbf{P}$ that allow for uniformly valid inference on the empirical process. Unfortunately, finding analogous restrictions on $\mathbf{P}$ for the problems we consider in this paper is very challenging, and we therefore leave it for future work-see Remark 3.1.

\section{MAIN RESULTS}

In this section, we show, for each of the three hypothesis testing problems we consider, that the power of any test against any alternative is always bounded above by the size of the test; that is, we show that (3) holds. As mentioned previously, these results further imply that any test that controls asymptotic size will have trivial asymptotic power against any alternative; that is, they imply (4).

\subsection{Testing Completeness}

We begin by establishing the nonexistence of nontrivial tests for completeness conditions. Toward this end, we first need to introduce additional notation and formally define $L^{q}$-completeness. Let $V_{i}=\left(X_{i}, Z_{i}\right) \in \mathbf{R}^{d_{x}} \times \mathbf{R}^{d_{z}}$ be random variables distributed according to $P \in \mathbf{P}$. For $Z_{i}=\left(Z_{i}^{(1)}, Z_{i}^{(2)}\right)$, with the subvector $Z_{i}^{(1)}$ possibly empty, let $W_{i}=\left(X_{i}, Z_{i}^{(1)}\right) \in \mathbf{R}^{d_{w}}$, and for $\Theta(P)$ a set of measurable functions from $\mathbf{R}^{d_{w}}$ to $\mathbf{R}$, consider the condition on $P$ given by

$$
E_{P}\left[\theta\left(W_{i}\right) \mid Z_{i}\right]=0 P \text {-a.s. for } \theta \in \Theta(P) \quad \Longrightarrow \quad \theta\left(W_{i}\right)=0 P \text {-a.s. }
$$

For $1 \leq q \leq \infty$, the distribution $P$ is said to be $L^{q}$-complete with respect to $W$ given $Z$ if condition (6) holds with $\Theta(P)=L^{q}\left(P_{W}\right)$. Here, $P_{W}$ denotes the marginal distribution of $W$ under $P$ and $L^{q}\left(P_{W}\right)$ denotes the set of measurable functions from $\mathbf{R}^{d_{w}}$ to $\mathbf{R}$ with finite $\|\cdot\|_{L^{q_{\left(P_{W}\right)}}}$ semi-norm—see the Appendix for definitions. For the special cases in which $q=1$ or $q=\infty, P$ is sometimes simply said to be complete with respect to $W$ given $Z$ or bounded complete with 
respect to $W$ given $Z$, respectively. See d'Haultfoeuille (2011) and Andrews (2011) for further discussion on these conditions.

As emphasized by Lemma 2.1, the definition of the set of possible distributions $\mathbf{P}$ plays a fundamental role in setting up the hypothesis testing problem. In our analysis, we restrict attention to sets of measures $\mathbf{P}$ that have a common dominating measure. Specifically, letting $\mathbf{M}_{x, z}$ denote the set of all Borel probability measures on $\mathbf{R}^{d_{x}} \times \mathbf{R}^{d_{z}}$, and defining the set

$$
\mathbf{M}_{x, z}(\nu) \equiv\left\{P \in \mathbf{M}_{x, z}: P \ll \nu\right\},
$$

for some Borel measure $\nu$ on $\mathbf{R}^{d_{x}} \times \mathbf{R}^{d_{z}}$, we let $\mathbf{P}=\mathbf{M}_{x, z}(\nu)$. In this setting, we examine the testability of the null hypothesis that the completeness condition fails, and hence, for a given choice of $\Theta(P)$, we let

$$
\mathbf{P}_{1}=\mathbf{P} \backslash \mathbf{P}_{0}=\{P \in \mathbf{P}:(6) \text { holds under } P\} .
$$

Defining the null hypothesis in this manner is analogous to testing the null hypothesis of a failure of the rank condition in a linear specification-see Remark 3.3. For instance, in a simple model where $\left(Y_{i}, X_{i}, Z_{i}\right) \in \mathbf{R}^{3}$ are distributed according to $P$, and for some $\theta \in \mathbf{R}$ we have

$$
E_{P}\left[Z_{i}\left(Y_{i}-X_{i} \theta\right)\right]=0,
$$

the rank condition required for identification reduces to $E_{P}\left[X_{i} Z_{i}\right] \neq 0$. Thus, our setup is analogous to testing the null hypothesis $E_{P}\left[X_{i} Z_{i}\right]=0$ (rank deficiency) against the alternative hypothesis $E_{P}\left[X_{i} Z_{i}\right] \neq 0$ (rank condition holds). We note that, in this simple example, the null hypothesis that the rank condition holds $\left(E_{P}\left[X_{i} Z_{i}\right] \neq 0\right)$ is untestable under typical assumptions.

We will make use of the following assumptions in establishing our result:

ASSUMPTION 3.1: $\nu$ is a positive $\sigma$-finite Borel measure on $\mathbf{R}^{d_{x}} \times \mathbf{R}^{d_{z}}$.

ASSUMPTION 3.2: $\nu=\nu_{x} \times \nu_{z}$, where $\nu_{x}$ and $\nu_{z}$ are Borel measures on $\mathbf{R}^{d_{x}}$ and $\mathbf{R}^{d_{z}}$, respectively.

ASSUMPTION 3.3: The measure $\nu_{x}$ is atomless on $\mathbf{R}^{d_{x}}$.

Note that since we impose the requirement that $\mathbf{P}=\mathbf{M}_{x, z}(\nu)$ for some $\nu$ satisfying Assumptions 3.1-3.3, properties of $\nu$ translate into restrictions on $\mathbf{P}$. For instance, if $\nu$ has bounded support, then $\mathbf{P}=\mathbf{M}_{x, z}(\nu)$ implies that the support of $\left(X_{i}, Z_{i}\right)$ under $P$ is uniformly bounded in $P \in \mathbf{P}$. In particular, by choosing $\nu_{x}$ and $\nu_{z}$ to be the Lebesgue measure on $[0,1]^{d_{x}}$ and $[0,1]^{d_{z}}$, respectively, we may impose the requirement that the support of $\left(X_{i}, Z_{i}\right)$ under $P$ be contained in $[0,1]^{d_{x}} \times[0,1]^{d_{z}}$ for all $P \in \mathbf{P}$. See Hall and Horowitz (2005) and Horowitz and Lee (2007) for examples of the use of such an assumption. It is also worth emphasizing that while Assumption 3.2 imposes that $\nu$ be a product measure, the 
requirement that $\mathbf{P}=\mathbf{M}_{x, z}(\nu)$ for some such $\nu$ does not imply that each $P \in \mathbf{P}$ is itself of such form. On the other hand, the requirement that $\mathbf{P}=\mathbf{M}_{x, z}(\nu)$ for some $\nu$ satisfying Assumptions 3.2 and 3.3 does imply that $P\left\{X_{i} \neq Z_{i}\right\}>0$ for all $P \in \mathbf{P}$. Thus, our assumptions rule out $X_{i}=Z_{i} P$-a.s. for some $P \in \mathbf{P}$, in which case condition (6) would be trivially satisfied. Finally, we point out that if $d_{x}>1$, then Assumption 3.3 may be weakened to instead requiring that at least one component of $X_{i}$ has an atomless marginal measure. For ease of exposition, however, we impose the stronger than necessary requirement in Assumption 3.3.

Under Assumptions 3.1-3.3, we can now establish the nontestability of bounded completeness.

THEOREM 3.1: Suppose Assumptions 3.1, 3.2, and 3.3 hold. If $\mathbf{P}=\mathbf{M}_{x, z}(\nu)$, for $\mathbf{M}_{x, z}(\nu)$ as in (7), and $\mathbf{P}_{0}$ and $\mathbf{P}_{1}$ are as in (8) with $\Theta(P)=L^{\infty}\left(P_{W}\right)$, then, for any sequence of tests $\left\{\phi_{n}\right\}_{n=1}^{\infty}$,

$$
\sup _{P \in \mathbf{P}_{1}} E_{P n}\left[\phi_{n}\right] \leq \sup _{P \in \mathbf{P}_{0}} E_{P n}\left[\phi_{n}\right] \quad \text { for all } n \geq 1 .
$$

Theorem 3.1 establishes the nonexistence of nontrivial tests for bounded completeness. The result is proven by showing that the set of distributions for which bounded completeness holds $\left(\mathbf{P}_{1}\right)$ lies in the closure of the set of distributions for which bounded completeness fails $\left(\mathbf{P}_{0}\right)$ with respect to the Total Variation distance and then appealing to Lemma 2.1. It therefore follows that the conclusion of Theorem 3.1 continues to hold if $\Theta(P)$ instead satisfies $L^{\infty}\left(P_{W}\right) \subseteq \Theta(P)$. Any such modification only enlarges $\mathbf{P}_{0}$, and hence $\mathbf{P}_{1}$ continues to lie in the closure of $\mathbf{P}_{0}$ with respect to the Total Variation distance. In particular, by setting $\Theta(P)=L^{q}\left(P_{W}\right)$ for any $1 \leq q<\infty$, we are able to conclude from Theorem 3.1 that there exist no nontrivial tests of $L^{q}$-completeness conditions either. It is worth emphasizing, however, that these results do rely on setting $\mathbf{P}=\mathbf{M}_{x, z}(\nu)$ and having $L^{\infty}\left(P_{W}\right) \subseteq \Theta(P)$. Thus, Theorem 3.1 does not preclude the existence of nontrivial tests for completeness conditions under more demanding restrictions on $\Theta(P)$ or $\mathbf{P}$; see Remarks 3.1-3.3 and the discussion thereafter.

While we focus on the testability of $L^{q}$-completeness conditions due to their importance in the literature, it is worth noting that they are only necessary conditions for identification. Indeed, if we consider $P$ as a measure on $\mathbf{R} \times$ $\mathbf{R}^{d_{x}} \times \mathbf{R}^{d_{z}}$ instead, then $P_{X, Z}$ being $L^{q}$-complete with respect to $W$ given $Z$ does not guarantee existence of a solution (in $\theta \in L^{q}\left(P_{W}\right)$ ) to the equation

$$
E_{P}\left[Y_{i}-\theta\left(W_{i}\right) \mid Z_{i}\right]=0 \quad P \text {-a.s. }
$$

Rather, $P_{X, Z}$ being $L^{q}$-complete with respect to $W$ given $Z$ just ensures that if a solution to (11) does exist, then it must be unique-see Proposition 2.1 in Newey and Powell (2003). Thus, $P_{X, Z}$ being $L^{q}$-complete with respect to 
$W$ given $Z$ is only equivalent to identification under the additional assumption that (11) holds for some $\theta \in L^{q}\left(P_{W}\right)$. In this sense, completeness requirements are analogous to the rank condition in a linear specification, which is necessary, but not sufficient, for ensuring identification; for example, if $\left(Y_{i}, X_{i}, Z_{i}\right) \in \mathbf{R} \times \mathbf{R} \times \mathbf{R}^{2}$, then $E_{P}\left[X_{i} Z_{i}\right]$ being full rank does not guarantee the existence of solution (in $\theta \in \mathbf{R}$ ) to equation (9).

REMARK 3.1: In establishing Theorem 3.1, we construct, for each $P \in \mathbf{P}_{1}$, a sequence $\left\{P_{k}\right\}_{k=1}^{\infty}$ in $\mathbf{P}_{0}$ such that $\left\|P-P_{k}\right\|_{\mathrm{TV}} \rightarrow 0$ as $k \rightarrow \infty$, and then apply Lemma 2.1 to obtain the desired conclusion. This approach requires us to exhibit, for each $P_{k}$, a corresponding function $\theta_{k} \in \Theta\left(P_{k}\right)$ such that

$$
P_{k}\left\{\theta_{k}\left(W_{i}\right) \neq 0\right\}>0 \quad \text { and } \quad E_{P_{k}}\left[\theta_{k}\left(W_{i}\right) \mid Z_{i}\right]=0 \quad P_{k} \text {-a.s. }
$$

While the $\theta_{k}$ that appear in the proof are not differentiable everywhere, it is worth emphasizing that this is not an essential feature of the argument. For instance, if $\nu$ equals the restriction of the Lebesgue measure on $\mathbf{R}^{d_{x}+d_{z}}$ to a compact set $K \subset \mathbf{R}^{d_{x}+d_{z}}$ and $\nu\{K\}>0$, then the construction in Lemma 2.1 of Santos (2012) implies that $P_{k}$ may be chosen so that both $d P_{k} / d \nu$ and $\theta_{k}$ are polynomials of finite order. Therefore, no nontrivial tests exist even if $\Theta(P)$ is further restricted to be a smooth class of functions, such as a Hölder space. By rescaling $\theta_{k}$ appropriately, we may, in fact, even restrict $\Theta(P)$ to be a Hölder ball. Analogously, no nontrivial tests exist if, for some finite integer $p$, we restrict every $P \in \mathbf{P}$ to be such that $d P / d \nu$ is $p$-times continuously differentiable, nor if we impose explicit bounds on the supremum of the first $p$ derivatives of $d P / d \nu$ on $K$.

REMARK 3.2: Under additional restrictions, the requirement that $\nu_{x}$ be atomless in Assumption 3.3 may be relaxed to it being a mixture of an atomless and a discrete measure. However, the conclusion of Theorem 3.1 may not apply if $\nu_{x}$ is a purely discrete measure. For example, suppose that $\nu_{x}$ and $\nu_{z}$ have finite support $\left\{x_{1}, \ldots, x_{s}\right\}$ and $\left\{z_{1}, \ldots, z_{t}\right\}$. Let $\Pi(P)$ be the $s \times t$ matrix with entry $\Pi(P)_{j k}=P\left\{X_{i}=x_{j} \mid Z_{i}=z_{k}\right\}$. Theorem 2.4 in Newey and Powell (2003) fully characterizes $L^{1}$-completeness of $P$ with respect to $W$ given $Z$ in terms of rank conditions on submatrices of $\Pi(P)$. In this setting, nontrivial tests for $L^{1}$-completeness of $P$ with respect to $W$ given $Z$ can therefore be constructed using, for example, uniformly valid confidence regions for $\Pi(P)$. See Anderson (1967) and Romano and Wolf (2000) for relevant results about confidence regions for a univariate mean.

REMARK 3.3: In the context of identification of some linear, semiparametric models with endogeneity, full rank requirements on matrices arise instead of completeness conditions. Specifically,

$$
\mathbf{P}_{1}=\mathbf{P} \backslash \mathbf{P}_{0}=\left\{P \in \mathbf{P}: E_{P}\left[Z_{i} W_{i}^{\prime}\right] \text { has full rank }\right\} .
$$


Tests for this purpose have been proposed, among others, by Anderson (1951), Gill and Lewbel (1992), Cragg and Donald (1993, 1997), Robin and Smith (2000), and Kleibergen and Paap (2006). Contrary to the implications of Theorem 3.1, nontrivial tests that control asymptotic size do exist, for example, if the support of $\left(X_{i}, Z_{i}\right)$ under $P$ is bounded uniformly in $P \in \mathbf{P}$.

Remarks 3.2 and 3.3 illustrate two important features of the problem we consider. First, they highlight that, despite their conceptual similarities, completeness conditions and rank conditions in linear specifications differ with regard to their testability. Second, they show that nontrivial tests of completeness may exist under alternative specifications of $\mathbf{P}$. For example, when $\mathbf{P}$ is given by certain exponential families of distributions, such as the multivariate normal, the sets $\mathbf{P}_{0}$ and $\mathbf{P}_{1}$ in (8) can be characterized by the rank of appropriate matrices in a manner similar to (12); see Newey and Powell (2003, Theorem 2.3). Thus, completeness conditions are also testable under suitable parametric restrictions on $\mathbf{P}$. Such restrictions, however, are at odds with the nonparametric nature of the problems in which completeness assumptions are often invoked.

Of course, an assumption being testable should not be considered a necessary condition for its use. Many assumptions are routinely imposed in empirical analysis that are untestable, yet deemed reasonable on an alternative basis. As an example, consider the commonly used assumption that $P$ has bounded support. This assumption is, in fact, untestable when $\mathbf{P}=\mathbf{M}_{x, z}$. Nonetheless, assuming that $P$ has bounded support can often be a natural requirement in a number of economic applications, and thus be deemed reasonable despite it being untestable.

The nonexistence of nontrivial tests for completeness, however, does emphasize the value of alternative justifications for its use. Examples of such arguments include Andrews (2011), who showed that the set of distributions for which $L^{2}$-completeness fails has a property analogous to having zero Lebesgue measure on $\mathbf{R}^{d}$. Formally, Andrews (2011) showed that the set of distributions for which $L^{2}$-completeness fails is "shy" within a certain set of distributions. See Hunt, Sauer, and Yorke (1992) and Anderson and Zame (2001) for a formal definition of shyness. In related work, Chen et al. (2012) showed that a certain class of probability measures over conditional expectation operators assign zero probability to the set of operators for which completeness fails.

\subsection{Testing Identification}

In this section, we establish analogous results to Theorem 3.1 for two widely studied nonparametric models with endogeneity that impose conditional quantile independence restrictions. Due to their nonlinear nature, simple, global rank conditions such as completeness conditions are unavailable for these models, and it is for this reason that we directly examine tests for identification instead. 
Throughout this section, we let $V_{i}=\left(Y_{i}, X_{i}, Z_{i}\right) \in \mathbf{R} \times \mathbf{R}^{d_{x}} \times \mathbf{R}^{d_{z}}$ be random variables distributed according to $P \in \mathbf{P}$. As before, we let $Z_{i}=\left(Z_{i}^{(1)}, Z_{i}^{(2)}\right)$, with the subvector $Z_{i}^{(1)}$ possibly empty, and let $W_{i}=\left(X_{i}, Z_{i}^{(1)}\right)$. We will once again focus on sets of distributions $\mathbf{P}$ that are dominated by a common measure $\nu$. Thus, by analogy with the notation used in the preceding section, we let $\mathbf{M}_{y, x, z}$ be the set of all Borel probability measures on $\mathbf{R} \times \mathbf{R}^{d_{x}} \times \mathbf{R}^{d_{z}}$ and define

$$
\mathbf{M}_{y, x, z}(\nu) \equiv\left\{P \in \mathbf{M}_{y, x, z}: P \ll \nu\right\} .
$$

We will impose the following requirements on the dominating measure $\nu$ :

ASSUMPTION 3.4: $\nu$ is a positive $\sigma$-finite Borel measure on $\mathbf{R} \times \mathbf{R}^{d_{x}} \times \mathbf{R}^{d_{z}}$.

ASSUMPTION 3.5: $\nu=\nu_{y} \times \nu_{x} \times \nu_{z}$, where $\nu_{y}, \nu_{x}$, and $\nu_{z}$ are Borel measures on $\mathbf{R}, \mathbf{R}^{d_{x}}$, and $\mathbf{R}^{d_{z}}$, respectively.

Assumptions 3.4 and 3.5 are modifications of Assumptions 3.1 and 3.2 from the previous section to account for the fact that here the random variables take values in $\mathbf{R} \times \mathbf{R}^{d_{x}} \times \mathbf{R}^{d_{z}}$ rather than just $\mathbf{R}^{d_{x}} \times \mathbf{R}^{d_{z}}$. In the results of this section, we will impose the requirement that $\mathbf{P} \subseteq \mathbf{M}_{y, x, z}(\nu)$ for some $\nu$ satisfying Assumptions 3.3, 3.4, and 3.5. Properties of $\nu$, such as bounded support, will therefore still translate into restrictions on $\mathbf{P}$-see the discussion preceding Theorem 3.1. Finally, we note that if $d_{x}>1$, then, in each of the following two theorems, Assumption 3.3 may be weakened to requiring that at least one component of $X_{i}$ has an atomless marginal measure.

\subsubsection{Single Quantile Restriction Model}

The first model we consider is one where, for an outcome of interest $Y_{i}$, an endogenous variable $X_{i}$ and an instrumental variable $Z_{i}$, and each $P \in \mathbf{P}$, there is some $\theta \in \Theta(P)$ such that

$$
P\left\{Y_{i}-\theta\left(W_{i}\right) \leq 0 \mid Z_{i}\right\}=\tau \quad P \text {-a.s. }
$$

for some prespecified $\tau \in(0,1)$. Here, $\Theta(P)$ is a set of measurable functions from $\mathbf{R}^{d_{w}}$ to $\mathbf{R}$, often set to equal $L^{q}\left(P_{W}\right)$ for some $1 \leq q \leq \infty$. We examine the testability of identification under the maintained assumption that $P$ indeed satisfies (14) for some $\theta \in \Theta(P)$. Thus, we define $\mathbf{P}$ to be

$$
\mathbf{P}=\left\{P \in \mathbf{M}_{y, x, z}(\nu): \exists \theta \in \Theta(P) \text { s.t. (14) holds under } P\right\} .
$$

By analogy with our analysis of the testability of completeness conditions, we let the null hypothesis be that identification fails in (14), and the alternative hypothesis be that it holds. Thus, we let

$$
\mathbf{P}_{1}=\mathbf{P} \backslash \mathbf{P}_{0}=\{P \in \mathbf{P}: \exists ! \theta \in \Theta(P) \text { s.t. (14) holds under } P\},
$$


where uniqueness of $\theta \in \Theta(P)$ is understood to be up to sets of measure zero under $P$.

The next result shows that no nontrivial tests of identification exist in models defined by (14).

THEOREM 3.2: Let Assumptions 3.3, 3.4, and 3.5 hold. Let $\mathbf{M}_{y, x, z}(\nu)$ be as in (13) and $\mathbf{P}$ be as in (15) with $\Theta(P)=L^{\infty}\left(P_{W}\right)$. If $\mathbf{P}_{0}$ and $\mathbf{P}_{1}$ are as in (16), then, for any sequence of tests $\left\{\phi_{n}\right\}_{n=1}^{\infty}$,

$$
\sup _{P \in \mathbf{P}_{1}} E_{P^{n}}\left[\phi_{n}\right] \leq \sup _{P \in \mathbf{P}_{0}} E_{P^{n}}\left[\phi_{n}\right] \text { for all } n \geq 1 .
$$

In establishing Theorem 3.2, we show that, for every $P \in \mathbf{P}_{1}$, there exists a sequence $\left\{P_{k}\right\}_{k=1}^{\infty}$ in $\mathbf{P}_{0}$ such that $\left\|P-P_{k}\right\|_{\mathrm{TV}} \rightarrow 0$ as $k \rightarrow \infty$. Our construction does not exploit the fact that $P \in \mathbf{P}_{1}$, but rather just the fact that $P \in \mathbf{M}_{y, x, z}(\nu)$. It follows that not only $\mathbf{P}_{1}$ lies in the closure of $\mathbf{P}_{0}$ with respect to the Total Variation metric, but that $\mathbf{M}_{y, x, z}(\nu)$ does as well. As a result, the conclusion of Theorem 3.2 continues to hold if we instead set $\Theta(P)=L^{q}\left(P_{W}\right)$ for any $1 \leq q<\infty$. It is worth noting that, in contrast to the setting of Theorem 3.1, here letting $\Theta(P)=L^{q}\left(P_{W}\right)$ for $1 \leq q<\infty$ enlarges $\mathbf{P}$ itself, and so potentially enlarges not only $\mathbf{P}_{0}$, but also $\mathbf{P}_{1}$. As in Theorem 3.1, however, it is important to emphasize that Theorem 3.2 does not preclude the testability of identification under alternative specifications of $\mathbf{P}$. For instance, Theorem 3.2 does not apply when $X_{i}$ has discrete support under $\nu_{x}$-a setting prominently studied in Chernozhukov and Hansen (2005).

REMARK 3.4: In establishing that $\mathbf{P}_{1}$ lies in the closure of $\mathbf{P}_{0}$ with respect to the Total Variation distance, we construct, for each $P \in \mathbf{P}_{1}$, a sequence $\left\{P_{k}\right\}_{k=1}^{\infty}$ in $\mathbf{P}_{0}$ such that $\left\|P-P_{k}\right\|_{\mathrm{TV}} \rightarrow 0$ as $k \rightarrow \infty$, and for each $k$, there exist $\theta_{k}^{(1)}$ and $\theta_{k}^{(2)}$ in $\Theta\left(P_{k}\right)$ that differ not only on a set with positive probability under $P_{k}$, but in the stronger sense of

$$
E_{P_{k}}\left[\left(1\left\{Y_{i} \leq \theta_{k}^{(1)}\left(W_{i}\right)\right\}-1\left\{Y_{i} \leq \theta_{k}^{(2)}\left(W_{i}\right)\right\}\right)^{2}\right]>0,
$$

while still satisfying

$$
P_{k}\left\{Y_{i} \leq \theta_{k}^{(1)}\left(W_{i}\right) \mid Z_{i}\right\}=P_{k}\left\{Y_{i} \leq \theta_{k}^{(2)}\left(W_{i}\right) \mid Z_{i}\right\}=\tau \quad P_{k} \text {-a.s. }
$$

This feature of the proof is noteworthy because it may still be the case that $1\left\{Y_{i} \leq \theta_{k}^{(1)}\left(W_{i}\right)\right\}=1\left\{Y_{i} \leq \theta_{k}^{(2)}\left(W_{i}\right)\right\} P_{k}$-a.s. for functions $\theta_{k}^{(1)}$ and $\theta_{k}^{(2)}$ that differ with positive probability under $P_{k}$. 


\subsubsection{Nonseparable Model}

The final model we consider is closely related to the single quantile independence model in (14). Specifically, for an outcome of interest $Y_{i}$, an endogenous variable $X_{i}$, and an instrumental variable $Z_{i}$, we now consider a setting in which, for each $P \in \mathbf{P}$, there is some $\theta \in \Theta(P)$ such that

$$
P\left\{Y_{i}-\theta\left(W_{i}, \tau\right) \leq 0 \mid Z_{i}\right\}=\tau \quad P \text {-a.s. for all } \tau \in(0,1) .
$$

Here, $\Theta(P)$ denotes a set of measurable functions $\theta: \mathbf{R}^{d_{w}} \times[0,1] \rightarrow \mathbf{R}$ such that $P$-a.s. the function $\tau \rightarrow \theta\left(W_{i}, \tau\right)$ is strictly increasing. Often, boundedness restrictions are imposed on $\theta$, so, for $\mathbf{T}$ the set of all measurable functions $\theta: \mathbf{R}^{d_{w}} \times[0,1] \rightarrow \mathbf{R}, \Theta(P)$ is set to equal

$$
\begin{aligned}
\mathbf{T}(P) \equiv & \left\{\theta \in \mathbf{T}: \tau \mapsto \theta\left(W_{i}, \tau\right) \text { is strictly increasing } P\right. \text {-a.s. and } \\
& \left.\sup _{0 \leq \tau \leq 1}\|\theta(\cdot, \tau)\|_{L^{\infty}(P)}<\infty\right\} .
\end{aligned}
$$

We consider the problem of testing for identification under the maintained assumption that $P$ indeed satisfies (18) for some $\theta \in \Theta(P)$. We therefore define $\mathbf{P}$ to be given by

$$
\mathbf{P}=\left\{P \in \mathbf{M}_{y, x, z}(\nu): \exists \theta \in \Theta(P) \text { s.t. (18) holds under } P\right\} .
$$

Following the hypothesis problem studied in Theorem 3.2, we examine the testability of the null hypothesis that $P \in \mathbf{P}$ is such that identification fails in (18). Formally, we define

$$
\mathbf{P}_{1}=\mathbf{P} \backslash \mathbf{P}_{0}=\{P \in \mathbf{P}: \exists ! \theta \in \Theta(P) \text { s.t. (18) holds under } P\},
$$

where uniqueness of $\theta \in \Theta(P)$ is understood to mean that if $\theta_{1} \in \Theta(P)$ and $\theta_{2} \in \Theta(P)$ both satisfy (18), then $P\left\{\theta_{1}\left(W_{i}, \tau\right)=\theta_{2}\left(W_{i}, \tau\right)\right\}=1$ for all $\tau \in(0,1)$.

It is worth noting that if (18) holds for a single, fixed $\tau \in(0,1)$, then this model is equivalent to the model in (14) with $\theta\left(W_{i}\right)=\theta\left(W_{i}, \tau\right)$. As a result of this connection between models (14) and (18), it is perhaps to be expected that the conclusion of Theorem 3.2 extends to the present setting. The following result establishes this point, showing that under commonly used restrictions for $\mathbf{P}$, no nontrivial tests of identification exist in models defined by (18) even if we impose that the function $\tau \mapsto \theta\left(W_{i}, \tau\right)$ be strictly increasing $P$-a.s.

THEOREM 3.3: Let Assumptions 3.3, 3.4, and 3.5 hold. Let $\mathbf{M}_{y, x, z}(\nu)$ be as in (13) and $\mathbf{P}$ be as in (19) with $\Theta(P)=\mathbf{T}(P)$. If $\mathbf{P}_{0}$ and $\mathbf{P}_{1}$ are as in (20), then, for any sequence of tests $\left\{\phi_{n}\right\}_{n=1}^{\infty}$,

$$
\sup _{P \in \mathbf{P}_{1}} E_{P^{n}}\left[\phi_{n}\right] \leq \sup _{P \in \mathbf{P}_{0}} E_{P^{n}}\left[\phi_{n}\right] \quad \text { for all } n \geq 1 .
$$


As in Theorem 3.2, our proof implies that not only $\mathbf{P}_{1}$ lies in the closure of $\mathbf{P}_{0}$ with respect to the Total Variation metric, but that $\mathbf{M}_{y, x, z}(\nu)$ does as well. It therefore follows that the conclusion of Theorem 3.3 continues to hold if we instead set $\Theta(P)$ to be given by

$$
\begin{aligned}
\Theta(P)= & \left\{\theta \in \mathbf{T}: \tau \mapsto \theta\left(W_{i}, \tau\right) \text { is strictly increasing } P\right. \text {-a.s. and } \\
& \left.\sup _{0 \leq \tau \leq 1}\|\theta(\cdot, \tau)\|_{L^{q}(P)}<\infty\right\}
\end{aligned}
$$

for some $1 \leq q<\infty$. It is also worth noting that the fact that $\mathbf{P}_{1}$ lies in the closure of $\mathbf{P}_{0}$ with respect to the Total Variation metric is established by constructing sequences $\left\{P_{k}\right\}_{k=1}^{\infty}$ in $\mathbf{P}_{0}$ such that, for each $k$, there exist functions $\theta_{k}^{(1)}$ and $\theta_{k}^{(2)}$ in $\Theta\left(P_{k}\right)$ satisfying

$$
E_{P_{k}}\left[\left(1\left\{Y_{i} \leq \theta_{k}^{(1)}\left(W_{i}, \tau\right)\right\}-1\left\{Y_{i} \leq \theta_{k}^{(2)}\left(W_{i}, \tau\right)\right\}\right)^{2}\right]>0
$$

for all $\tau \in(0,1)$. Thus, the functions $\theta_{k}^{(1)}(\cdot, \tau)$ and $\theta_{k}^{(2)}(\cdot, \tau)$ differ for every $\tau$ not just on a set with positive probability under $P_{k}$, but in the stronger sense of Remark 3.4.

\section{CONCLUSION}

This paper shows that, under commonly imposed restrictions on the distribution of the data, no nontrivial tests for completeness or identification conditions exist in some prominent nonparametric models with endogeneity. Whether testability can be restored under appealing restrictions on either $\Theta(P)$ or $\mathbf{P}$ remains an important and challenging open question.

Two constructive points follow from our results, which we believe have potentially important implications for future research. First, having established the impossibility of producing empirical evidence in favor of completeness or identification conditions, our results emphasize the value of alternative arguments for their plausibility. Recent work that addresses this problem includes Andrews (2011) and Chen, Chernozhukov, Lee, and Newey (2012), who argued in favor of completeness conditions on the basis of genericity arguments. Second, our analysis highlights the significance of both developing inferential methods that are robust to partial identification and comparing their performance to nonrobust inferential approaches. For instance, it would be important to understand which inferential method is preferable when identification is believed to hold. We hope the results and arguments in this paper provide motivation for addressing these challenges in future research.

\section{APPENDIX}

Throughout the appendix, we employ the following notation, not necessarily introduced in the text: 
$A \triangle B$ For two sets $A$ and $B, A \triangle B \equiv(A \backslash B) \cup(B \backslash A)$. of $A$.

$\mathcal{B}(A)$ For a Borel set $A$, the $\sigma$-algebra generated by all open subsets

$\|\cdot\|_{L^{q}(\lambda)}$ For $1 \leq q<\infty$, a measure $\lambda$, and measurable function $f,\|f\|_{L^{q}(\lambda)}^{q} \equiv$ $\int|f(u)|^{q} \lambda(d u)$.

$\|\cdot\|_{L^{\infty}(\lambda)}$ For a measure $\lambda$ and measurable function $f,\|f\|_{L^{\infty}(\lambda)} \equiv$ esssup $|f(u)|$, where the essential supremum is defined as ess $\sup |f(u)| \equiv$ $\inf \{M \in \mathbf{R}: \lambda\{u:|f(u)|>M\}=0\}$.

$L^{q}(\lambda)$ For $1 \leq q \leq \infty$ and a measure $\lambda, L^{q}(\lambda)$ is the space of measurable functions $f$ with finite semi-norm $\|f\|_{L^{q}(\lambda)}$.

LEMMA A.1: Let $A \subseteq \mathbf{R}^{d}$ be a Borel set, $\mathcal{B}(A)$ the $\sigma$-algebra generated by all open subsets of $A$, and $\lambda$ an atomless positive Borel measure on $\mathbf{R}^{d}$ satisfying $0<$ $\lambda\{A\}<\infty$. Then, there exists a map $\bar{B}:[0,1] \rightarrow \mathcal{B}(A)$ such that: (i) $\bar{B}(0)=\emptyset$ and $\bar{B}(1)=A$, (ii) $\bar{B}(\tau) \subseteq \bar{B}\left(\tau^{\prime}\right)$ for all $0 \leq \tau \leq \tau^{\prime} \leq 1$, and (iii) $\lambda\{\bar{B}(\tau)\}=\tau \lambda\{A\}$ for all $\tau \in[0,1]$. Additionally, there is $\tilde{B}:[0,1] \rightarrow \mathcal{B}(A)$ satisfying properties (i)-(iii) and such that $\lambda\{\bar{B}(\tau) \triangle \tilde{B}(\tau)\}>0$ for all $\tau \in(0,1)$.

ProOF: Without loss of generality, we assume $\lambda\{A\}=1$; otherwise, we may just renormalize. Let $\mu$ denote the Lebesgue measure on $\mathbf{R}$, and $\mathcal{B}([0,1])$ the $\sigma$-algebra generated by all open subsets of $[0,1]$. For any $U_{1}, U_{2} \in \mathcal{B}(A)$, define the equivalence relation $U_{1} \sim U_{2}$ if $\lambda\left\{U_{1} \triangle U_{2}\right\}=0$, and denote the set of resulting equivalence classes by $E_{\lambda}$. Similarly, denote by $E_{\mu}$ the equivalence classes on $\mathcal{B}([0,1])$ generated by $\mu$. Next, observe that since $\lambda$ is a Borel measure on $\mathbf{R}^{d}$, Theorem 7.1.7 in Bogachev (2007b) implies $\lambda$ is Radon, and hence also separable by Proposition 7.14.12(ii) in Bogachev (2007b). It therefore follows from Theorem 9.3.4 in Bogachev (2007b) that $\left(E_{\lambda}, \lambda\right)$ is isomorphic to $\left(E_{\mu}, \mu\right)$, that is, there exists a one-to-one mapping $\Gamma: E_{\lambda} \rightarrow E_{\mu}$ such that

$$
\begin{aligned}
& \mu\left\{\Gamma\left(V_{1}\right)\right\}=\lambda\left\{V_{1}\right\}, \\
& \Gamma\left(V_{1} \backslash V_{2}\right)=\Gamma\left(V_{1}\right) \backslash \Gamma\left(V_{2}\right), \quad \Gamma\left(V_{1} \cup V_{2}\right)=\Gamma\left(V_{1}\right) \cup \Gamma\left(V_{2}\right)
\end{aligned}
$$

for any $V_{1}, V_{2} \in E_{\lambda}$, and where the operations on elements of $E_{\mu}$ and $E_{\lambda}$ are defined as the respective operations on arbitrary representatives of the equivalence classes. Next, define a map $B:(0,1) \rightarrow \mathcal{B}(A)$ satisfying $B(\tau) \in$ $\Gamma^{-1}([0, \tau])$ for any $\tau \in(0,1)$, and note that, by (22), $B$ satisfies $\lambda\{B(\tau)\}=\tau$ for all $\tau \in(0,1)$. The map $B$, however, may not be monotonic, and for this reason we modify it by defining $\bar{B}:[0,1] \rightarrow \mathcal{B}(A)$ to be given by $\bar{B}(0)=\emptyset, \bar{B}(1)=A$ and for any $\tau \in(0,1)$ by

$$
\bar{B}(\tau) \equiv\left[B(\tau) \cup\left\{\bigcup_{0<\sigma<\tau, \sigma \in \mathbf{Q}} B(\sigma)\right\}\right] \cap\left\{\bigcap_{1>\sigma>\tau, \sigma \in \mathbf{Q}} B(\sigma)\right\},
$$


where $\mathbf{Q}$ are the rational numbers. It follows by construction that $\bar{B}$ then satisfies properties (i) and (ii). Moreover, for any $\tau \in(0,1)$, let $\left\{a_{i}(\tau)\right\}_{i=1}^{\infty}=$ $\mathbf{Q} \cap(0, \tau)$, and note that, by $(22)$, we have

$$
\begin{aligned}
\lambda\{\bar{B}(\tau) \backslash B(\tau)\} & \leq \lim _{n \rightarrow \infty} \lambda\left\{\left\{\bigcup_{i=1}^{n} B\left(a_{i}(\tau)\right)\right\} \backslash B(\tau)\right\} \\
& =\lim _{n \rightarrow \infty} \mu\left\{\left\{\bigcup_{i=1}^{n}\left[0, a_{i}(\tau)\right]\right\} \backslash[0, \tau]\right\}=0 .
\end{aligned}
$$

Similarly, letting $\left\{b_{i}(\tau)\right\}_{i=1}^{\infty}=\mathbf{Q} \cap(\tau, 1)$, we also obtain by monotone convergence that

$$
\begin{aligned}
\lambda\{B(\tau) \backslash \bar{B}(\tau)\} & =\lim _{n \rightarrow \infty} \lambda\left\{B(\tau) \backslash\left\{\bigcap_{i=1}^{n} B\left(b_{i}(\tau)\right)\right\}\right\} \\
& =\lim _{n \rightarrow \infty} \mu\left\{[0, \tau] \backslash\left\{\bigcap_{i=1}^{n}\left[0, b_{i}(\tau)\right]\right\}\right\}=0 .
\end{aligned}
$$

We conclude from (24) and (25) that $\lambda\{\bar{B}(\tau)\}=\lambda\{B(\tau)\}$ for all $\tau \in(0,1)$. Since $\lambda\{B(\tau)\}=\mu\{[0, \tau]\}=\tau$ for all $\tau \in(0,1), \bar{B}(0)=\emptyset$ and $\bar{B}(1)=A$, it follows that $\bar{B}$ satisfies property (iii) as well. ${ }^{2}$

To establish the second claim of the lemma, pointwise define $\tilde{B}:[0,1] \rightarrow$ $\mathcal{B}(A)$ by

$$
\tilde{B}(\tau) \equiv A \backslash \bar{B}(1-\tau) .
$$

It is then immediate that $\tilde{B}(0)=\emptyset$ and $\tilde{B}(1)=A$, while $\lambda\{\bar{B}(\tau)\}=\tau$ for all $\tau \in[0,1]$ additionally yields that

$$
\lambda\{\tilde{B}(\tau)\}=\lambda\{A \backslash \bar{B}(1-\tau)\}=1-(1-\tau)=\tau
$$

for all $\tau \in[0,1]$. Furthermore, for any $0 \leq \tau \leq \tau^{\prime} \leq 1$, note that $\tau \leq \tau^{\prime}$ implies $\bar{B}\left(1-\tau^{\prime}\right) \subseteq \bar{B}(1-\tau)$, and therefore

$$
\tilde{B}(\tau)=A \backslash \bar{B}(1-\tau) \subseteq A \backslash \bar{B}\left(1-\tau^{\prime}\right)=\tilde{B}\left(\tau^{\prime}\right) .
$$

Thus, from (27) and (28), we obtain that $\tilde{B}:[0,1] \rightarrow \mathcal{B}(A)$ indeed satisfies properties (i)-(iii). To conclude, note that monotonicity of $\bar{B}$ implies

\footnotetext{
${ }^{2}$ We would like to thank an anonymous referee for suggesting this method of proof.
} 
$(A \backslash \bar{B}(1-\tau)) \backslash \bar{B}(\tau)=A \backslash \bar{B}(\max \{\tau, 1-\tau\})$, and hence

$$
\begin{aligned}
\lambda\{\tilde{B}(\tau) \triangle \bar{B}(\tau)\} & \geq \lambda\{\tilde{B}(\tau) \backslash \bar{B}(\tau)\} \\
& =\lambda\{A \backslash \bar{B}(\max \{\tau, 1-\tau\})\}=(1-\max \{\tau, 1-\tau\}) .
\end{aligned}
$$

Therefore, it follows from (29) that $\lambda\{\tilde{B}(\tau) \triangle \bar{B}(\tau)\}>0$ for all $\tau \in(0,1)$.Q.E.D.

LEMMA A.2: Let $Q$ be a Borel probability measure on $\mathbf{R} \times \mathbf{R}^{d_{x}} \times \mathbf{R}^{d_{z}}$ satisfying $Q \ll \lambda$ for $\lambda$ a $\sigma$-finite positive Borel measure on $\mathbf{R} \times \mathbf{R}^{d_{x}} \times \mathbf{R}^{d_{z}}$, and let $f \equiv d Q / d \lambda$. Then, for each $k \in \mathbb{N}$, there exists a positive real number $M_{k}, a$ natural number $K_{k}$, collections $\left\{A_{i k}\right\}_{i=1}^{K_{k}},\left\{B_{j k}\right\}_{j=1}^{K_{k}},\left\{C_{l k}\right\}_{l=1}^{K_{k}}$ that are partitions of $\left[-M_{k}, M_{k}\right],\left[-M_{k}, M_{k}\right]^{d_{x}}$, and $\left[-M_{k}, M_{k}\right]^{d_{z}}$, respectively, and a set of positive real numbers $\left\{\pi_{i j l k}\right\}_{1 \leq i, j, l \leq K_{k}}$ such that the sequence of functions $\left\{f_{k}\right\}_{k=1}^{\infty}$ defined by

$$
f_{k}(y, x, z)=\sum_{1 \leq i, j, l \leq K_{k}} \pi_{i j l k} 1\left\{(y, x, z) \in A_{i k} \times B_{j k} \times C_{l k}\right\}
$$

satisfies: (i) $f_{k} \geq 0$ for all $k$, (ii) $\int f_{k} d \lambda=1$ for all $k$, and (iii) $\left\|f_{k}-f\right\|_{L^{1}(\lambda)} \rightarrow 0$ as $k \rightarrow \infty$.

Proof: Note $f \geq 0$ and $f \in L^{1}(\lambda)$. Since $\lambda$ is a Borel measure, it is also regular by Theorem 7.1.7 in Bogachev (2007b), and hence Theorem 13.9 in Aliprantis and Border (2006) implies there is a sequence $\left\{f_{k}^{\star}\right\}_{k=1}^{\infty}$ of continuous, compactly supported functions such that $f_{k}^{\star} \geq 0$ for all $k$ and

$$
\left\|f_{k}^{\star}-f\right\|_{L^{1}(\lambda)} \rightarrow 0 \quad \text { as } \quad k \rightarrow \infty .
$$

Let $\Omega_{k} \subset \mathbf{R} \times \mathbf{R}^{d_{x}} \times \mathbf{R}^{d_{z}}$ be the compact support of $f_{k}^{\star}$ and select $M_{k}>0$ sufficiently large so that $\Omega_{k} \subseteq\left[-M_{k}, M_{k}\right]^{1+d_{x}+d_{z}}$. Additionally, select $0<\xi_{k} \downarrow 0$ so that $\lim _{k \rightarrow \infty} \xi_{k} \lambda\left\{\left[-M_{k}, M_{k}\right]^{1+d_{x}+d_{z}}\right\}=0$, and notice that $f_{k}^{\star}$ being uniformly continuous on $\left[-M_{k}, M_{k}\right]^{1+d_{x}+d_{z}}$ implies that there exist partitions $\left\{A_{i k}\right\}_{i=1}^{K_{k}}$, $\left\{B_{j k}\right\}_{j=1}^{K_{k}}$, and $\left\{C_{l k}\right\}_{l=1}^{K_{k}}$ of $\left[-M_{k}, M_{k}\right],\left[-M_{k}, M_{k}\right]^{d_{x}}$, and $\left[-M_{k}, M_{k}\right]^{d_{z}}$, such that

$$
\max _{1 \leq i, j, l \leq K_{k}} \sup _{(y, x, z) \in A_{i k} \times B_{j k} \times C_{l k}} \sup _{(\tilde{y}, \tilde{x}, \tilde{z}) \in A_{i k} \times B_{j k} \times C_{l k}}\left|f_{k}^{\star}(y, x, z)-f_{k}^{\star}(\tilde{y}, \tilde{x}, \tilde{z})\right| \leq \xi_{k} .
$$

Letting $S_{i j l k} \equiv A_{i k} \times B_{j k} \times C_{l k}$ for $1 \leq i, j, l \leq K_{k}$, we then pointwise define the functions

$$
\begin{aligned}
& \tilde{f}_{k}(y, x, z) \equiv \sum_{1 \leq i, j, l \leq K_{k}} \tilde{\pi}_{i j l k} 1\left\{(y, x, z) \in S_{i j l k}\right\}, \\
& \tilde{\pi}_{i j l k} \equiv \sup _{(y, x, z) \in S_{i j l k}} f_{k}^{\star}(y, x, z) .
\end{aligned}
$$


By construction, $\tilde{\pi}_{i j l k} \geq 0$ for all $1 \leq i, j, l \leq K_{k}$ and all $k$, and hence $\tilde{f}_{k} \geq 0$. Moreover, since $f_{k}^{\star}, \tilde{f}_{k}$ vanish outside $\left[-M_{k}, M_{k}\right]^{1+d_{x}+d_{z}}$, and $\left\{S_{i j k l}\right\}_{1 \leq i, j, l \leq K_{k}}$ is a partition of $\left[-M_{k}, M_{k}\right]^{1+d_{x}+d_{z}}$, equations (32) and (33) imply

$$
\sup _{(y, x, z) \in \mathbf{R}^{1+d_{x}+d_{z}}}\left|f_{k}^{\star}(y, x, z)-\tilde{f}_{k}(y, x, z)\right| \leq \xi_{k} .
$$

Thus, we obtain

$$
\begin{aligned}
\lim _{k \rightarrow \infty}\left\|f_{k}^{\star}-\tilde{f}_{k}\right\|_{L^{1}(\lambda)} & =\lim _{k \rightarrow \infty} \int_{\left[-M_{k}, M_{k}\right]^{1+d_{x}+d_{z}}}\left|f_{k}^{\star}-\tilde{f}_{k}\right| d \lambda \\
& \leq \lim _{k \rightarrow \infty} \xi_{k} \lambda\left\{\left[-M_{k}, M_{k}\right]^{1+d_{x}+d_{z}}\right\}=0,
\end{aligned}
$$

since $\xi_{k} \downarrow 0$ satisfies $\lim _{k \rightarrow \infty} \xi_{k} \lambda\left\{\left[-M_{k}, M_{k}\right]^{1+d_{x}+d_{z}}\right\}=0$. Finally, let $f_{k} \equiv$ $\tilde{f}_{k} /\left\|\tilde{f}_{k}\right\|_{L^{1}(\lambda)}$ and note that properties (i) and (ii) follow, while (30) holds with $\pi_{i j l k}=\tilde{\pi}_{i j k} /\left\|\tilde{f}_{k}\right\|_{L^{1}(\lambda)}$. Moreover, since $\left\|\tilde{f}_{k}-f\right\|_{L^{1}(\lambda)} \rightarrow 0$ as $k \rightarrow \infty$ by (31) and (34), it follows that $\left\|\tilde{f}_{k}\right\|_{L^{1}(\lambda)} \rightarrow 1$, and thus

$$
\begin{aligned}
\left\|f_{k}-f\right\|_{L^{1}(\lambda)} & \leq \frac{\left\|\tilde{f}_{k}-f\right\|_{L^{1}(\lambda)}}{\left\|\tilde{f}_{k}\right\|_{L^{1}(\lambda)}}+\left|1-\frac{1}{\left\|\tilde{f}_{k}\right\|_{L^{1}(\lambda)}}\right| \times\|f\|_{L^{1}(\lambda)} \\
& =o(1) \quad \text { as } \quad k \rightarrow \infty,
\end{aligned}
$$

which verifies property (iii), and hence the claim of the lemma follows. Q.E.D.

Proof of Lemma 2.1: Fix $P \in \mathbf{P}_{1}$ and let $\left\{P_{k}\right\}_{k=1}^{\infty}$ satisfy $\left\|P_{k}-P\right\|_{\mathrm{TV}} \rightarrow 0$ as $k \rightarrow \infty$ and $P_{k} \in \mathbf{P}_{0}$ for all $k$. Since $\left\|P_{k}-P\right\|_{\mathrm{TV}} \rightarrow 0$ as $k \rightarrow \infty$ implies $\left\|P_{k}^{n}-P^{n}\right\|_{\mathrm{TV}} \rightarrow \infty$ for any fixed $n \in \mathbb{N}$ (see (4.5) in Hoeffding and Wolfowitz (1958)), the result immediately follows from Theorem 1 in Romano (2004) applied to the sets $\mathbf{P}_{0, n} \equiv\left\{Q \in \bigotimes_{i=1}^{n} \mathbf{M}: Q=P^{n}\right.$ for some $\left.P \in \mathbf{P}_{0}\right\}$ and $\mathbf{P}_{1, n} \equiv$ $\left\{Q \in \bigotimes_{i=1}^{n} \mathbf{M}: Q=P^{n}\right.$ for some $\left.P \in \mathbf{P}_{1}\right\}$.

Q.E.D.

Proof of Theorem 3.1: Fix $P \in \mathbf{P}_{1}$ and let $f \equiv d P / d \nu$. By Assumption 3.1 and Lemma A.2 applied to $Q \equiv \delta_{0} \times P$ and $\lambda \equiv \delta_{0} \times \nu$ for $\delta_{0}$ a degenerate measure at 0 on $\mathbf{R}$, there is $\left\{f_{k}\right\}_{k=1}^{\infty}$ with

$$
\left\|f_{k}-f\right\|_{L^{1}(\nu)}=o(1) \quad \text { as } \quad k \rightarrow \infty,
$$

and in addition, for all $k$, each $f_{k}$ satisfies $f_{k} \geq 0, \int f_{k} d \nu=1$, and is a simple function of the form

$$
f_{k}(x, z)=\sum_{1 \leq j, l \leq K_{k}} \pi_{j l k} 1\left\{(x, z) \in S_{j l k}\right\} .
$$


Here, $S_{j l k}=B_{j k} \times C_{l k}$, and for some $M_{k}>0$, the collections $\left\{B_{j k}\right\}_{j=1}^{K_{k}}$ and $\left\{C_{l k}\right\}_{l=1}^{K_{k}}$ are partitions of $\left[-M_{k}, M_{k}\right]^{d_{x}}$ and $\left[-M_{k}, M_{k}\right]^{d_{z}}$, respectively. Therefore, defining

$$
P_{k}\{E\} \equiv \int_{E} f_{k} d \nu
$$

for all Borel measurable $E \subseteq \mathbf{R}^{d_{x}} \times \mathbf{R}^{d_{z}}$, it follows that $P_{k}$ is a probability measure with $P_{k} \ll \nu$, and hence $P_{k} \in \mathbf{P}=\mathbf{M}_{x, z}(\nu)$ for all $k$. Moreover, by (36), $\left\|P_{k}-P\right\|_{\mathrm{TV}} \rightarrow 0$ as $k \rightarrow \infty$.

In what follows, we aim to show that in fact $P_{k} \in \mathbf{P}_{0}$ for all $k$. Assumption 3.3 and Corollary 1.12.10 in Bogachev (2007a) imply that, for each $1 \leq j \leq K_{k}$, there exist Borel measurable sets $B_{j k}^{(1)}, B_{j k}^{(2)}$ such that $B_{j k}=B_{j k}^{(1)} \cup B_{j k}^{(2)}, B_{j k}^{(1)} \cap$ $B_{j k}^{(2)}=\emptyset$ and in addition satisfy

$$
\nu_{x}\left\{B_{j k}^{(1)}\right\}=\nu_{x}\left\{B_{j k}^{(2)}\right\}=\frac{1}{2} \nu_{x}\left\{B_{j k}\right\}
$$

Since $\left\{B_{j k}\right\}_{j=1}^{K_{k}}$ is a partition of $\left[-M_{k}, M_{k}\right]^{d_{x}}$ by Lemma A.2, we may define a function $\theta_{k}$ by

$$
\theta_{k}(x) \equiv \sum_{j=1}^{K_{k}}\left(1\left\{x \in B_{j k}^{(1)}\right\}-1\left\{x \in B_{j k}^{(2)}\right\}\right) .
$$

Note that $\theta_{k} \in L^{\infty}\left(P_{k}\right)$, and $\theta_{k} \neq 0 P_{k}$-a.s. due to (37), (40) and $B_{j k}^{(1)} \cap B_{j k}^{(2)}=\emptyset$ for all $1 \leq j \leq K_{k}$. Moreover, for any bounded $z \mapsto \psi(z)$, we obtain from (37), $f_{k}=d P_{k} / d \nu$, and Assumption 3.2:

$$
\begin{aligned}
& E_{P_{k}}\left[\psi\left(Z_{i}\right) \theta_{k}\left(X_{i}\right)\right] \\
& \quad=\sum_{1 \leq j, l \leq K_{k}} \pi_{j l k} \int_{C_{l k}} \int_{B_{j k}} \psi(z) \theta_{k}(x) \nu_{x}(d x) \nu_{z}(d z) \\
& \quad=\sum_{1 \leq j, l \leq K_{k}} \pi_{j l k}\left(\nu_{x}\left\{B_{j k}^{(1)}\right\}-\nu_{x}\left\{B_{j k}^{(2)}\right\}\right) \int_{C_{l k}} \psi(z) \nu_{z}(d z) \\
& \quad=0,
\end{aligned}
$$

where the final equality exploited (39). In particular, (41) must hold for $\psi(\cdot)=$ $E_{P_{k}}\left[\theta_{k}\left(X_{i}\right) \mid Z_{i}=\cdot\right]$, and hence we obtain, by the law of iterated expectations, that $E_{P_{k}}\left[\theta_{k}\left(X_{i}\right) \mid Z_{i}\right]=0 P_{k}$-a.s. Note that for each $k, \theta_{k}$ can be viewed as a function of $w=\left(x, z^{(1)}\right)$ that only depends on $x$, which together with (41) suffices for concluding that $P_{k} \in \mathbf{P}_{0}$ for all $k$. Hence, since $P \in \mathbf{P}_{1}$ was arbi- 
trary and $\left\|P_{k}-P\right\|_{\mathrm{TV}} \rightarrow 0$ as $k \rightarrow \infty$, the conclusion of the theorem follows by Lemma 2.1.

Q.E.D.

Proof of Theorem 3.2: Fix $P \in \mathbf{P}_{1}$ and let $f \equiv d P / d \nu$. As in the proof of Theorem 3.1, we begin by noting that Lemma A.2 and Assumption 3.4 imply that there is a sequence $\left\{f_{k}\right\}_{k=1}^{\infty}$ such that

$$
\left\|f_{k}-f\right\|_{L^{1}(\nu)}=o(1) \quad \text { as } \quad k \rightarrow \infty,
$$

and in addition, for all $k$, each $f_{k}$ satisfies $f_{k} \geq 0, \int f_{k} d \nu=1$, and is a simple function of the form

$$
f_{k}(y, x, z)=\sum_{1 \leq i, j, l \leq K_{k}} \pi_{i j l k} 1\left\{(y, x, z) \in S_{i j l k}\right\} .
$$

Here, $S_{i j l k}=A_{i k} \times B_{j k} \times C_{l k}$, and for some $M_{k}>0,\left\{A_{i k}\right\}_{i=1}^{K_{k}},\left\{B_{j k}\right\}_{j=1}^{K_{k}}$, and $\left\{C_{l k}\right\}_{l=1}^{K_{k}}$ form partitions of $\left[-M_{k}, M_{k}\right],\left[-M_{k}, M_{k}\right]^{d_{x}}$, and $\left[-M_{k}, M_{k}\right]^{d_{z}}$, respectively. Hence, defining

$$
P_{k}\{E\} \equiv \int_{E} f_{k} d \nu
$$

for all Borel measurable $E \subseteq \mathbf{R} \times \mathbf{R}^{d_{x}} \times \mathbf{R}^{d_{z}}$, we obtain a sequence of probability measure $\left\{P_{k}\right\}_{k=1}^{\infty}$ satisfying $\bar{P}_{k} \in \mathbf{M}_{y, x, z}(\nu)$ for all $k$, and $\left\|P_{k}-P\right\|_{\mathrm{TV}} \rightarrow 0$ as $k \rightarrow$ $\infty$ due to result (42).

We next aim to show that $P_{k} \in \mathbf{P}_{0}$ for all $k$. Toward this end, note that Assumption 3.3 and Lemma A.1 imply that there exist collections of sets $\left\{B_{j k}^{(1)}(\tau), B_{j k}^{(2)}(\tau)\right\}_{j=1}^{K_{k}}$ such that, for all $1 \leq j \leq K_{k}$,

$$
\nu_{x}\left\{B_{j k}^{(1)}(\tau)\right\}=\tau \nu_{x}\left\{B_{j k}\right\}, \quad \nu_{x}\left\{B_{j k}^{(2)}(\tau)\right\}=\tau \nu_{x}\left\{B_{j k}\right\},
$$

with $B_{j k}^{(m)}(\tau) \subseteq B_{j k}$ for $m \in\{1,2\}$, and $\nu_{x}\left\{B_{j k}^{(1)}(\tau) \triangle B_{j k}^{(2)}(\tau)\right\}>0$ for all $1 \leq j \leq K_{k}$ such that $\nu_{x}\left\{B_{j k}\right\}>0$. For $m \in\{1,2\}$, we may then define functions $\theta_{k}^{(m)}(\cdot, \tau)$ pointwise in $x$ by

$$
\theta_{k}^{(m)}(x, \tau) \equiv \sum_{j=1}^{K_{k}}\left(2 M_{k} 1\left\{x \in B_{j k}^{(m)}(\tau)\right\}-2 M_{k} 1\left\{x \in B_{j k} \backslash B_{j k}^{(m)}(\tau)\right\}\right),
$$

and note that $\theta_{k}^{(m)}(\cdot, \tau) \in L^{\infty}\left(P_{k}\right)$ for $m \in\{1,2\}$. Moreover, $Y_{i} \in\left[-M_{k}, M_{k}\right] P_{k^{-}}$ a.s., together with Assumption 3.5 and $f_{k}=d P_{k} / d \nu$, with $f_{k}$ as in (43), allows us to conclude that

$$
\begin{aligned}
& E_{P_{k}}\left[\left(1\left\{Y_{i} \leq \theta_{k}^{(1)}\left(X_{i}, \tau\right)\right\}-1\left\{Y_{i} \leq \theta_{k}^{(2)}\left(X_{i}, \tau\right)\right\}\right)^{2}\right] \\
& \quad=E_{P_{k}}\left[\left(1\left\{\theta_{k}^{(1)}\left(X_{i}, \tau\right)=2 M_{k}\right\}-1\left\{\theta_{k}^{(2)}\left(X_{i}, \tau\right)=2 M_{k}\right\}\right)^{2}\right]
\end{aligned}
$$




$$
\begin{aligned}
= & \sum_{1 \leq i, j, l \leq K_{k}} \pi_{i j l k} \nu_{y}\left\{A_{i k}\right\} \nu_{z}\left\{C_{l k}\right\} \\
& \times \int_{B_{j k}}\left(1\left\{\theta_{k}^{(1)}(x, \tau)=2 M_{k}\right\}-1\left\{\theta_{k}^{(2)}(x, \tau)=2 M_{k}\right\}\right)^{2} \nu_{x}(d x) \\
> & 0,
\end{aligned}
$$

where we exploited $\left(1\left\{\theta_{k}^{(1)}(x, \tau)=2 M_{k}\right\}-1\left\{\theta_{k}^{(2)}(x, \tau)=2 M_{k}\right\}\right)^{2}=1\{x \in$ $\left.B_{j k}^{(1)}(\tau) \triangle B_{j k}^{(2)}(\tau)\right\}$ for every $x \in B_{j k}$ and $\nu_{x}\left\{B_{j k}^{(1)}(\tau) \triangle B_{j k}^{(2)}(\tau)\right\}>0$ for some $1 \leq j \leq K_{k}$ due to $P_{k}$ having support $\left[-M_{k}, M_{k}\right]^{1+d_{x}+d_{z}}$ implying $P_{k}\left\{S_{i j l k}\right\}=$ $\pi_{i j l k} \nu\left\{S_{i j l k}\right\}>0$ for some $1 \leq i, j, l \leq K_{k}$.

We conclude from $(47)$ that $\theta_{k}^{(1)}(\cdot, \tau)$ and $\theta_{k}^{(2)}(\cdot, \tau)$ are distinct under $\|\cdot\|_{L^{\infty}\left(P_{k}\right)}$. Additionally, for $m \in\{1,2\}$, we have $1\left\{\theta_{k}^{(m)}(x, \tau)=2 M_{k}\right\}=1\{x \in$ $\left.B_{j k}^{(m)}(\tau)\right\}$ for every $x \in B_{j k}$ by (46), and hence $\nu_{x}\left\{\left\{\theta_{k}^{(m)}(x, \tau)=2 M_{k}\right\} \cap B_{j k}\right\}=$ $\nu_{x}\left\{B_{j k}^{(m)}(\tau)\right\}=\tau \nu_{x}\left\{B_{j k}\right\}$. It follows that, for any bounded $z \mapsto \psi(z)$,

$$
\begin{aligned}
E_{P_{k}} & {\left[\psi\left(Z_{i}\right)\left(1\left\{Y_{i} \leq \theta_{k}^{(m)}\left(X_{i}, \tau\right)\right\}-\tau\right)\right] } \\
= & \sum_{1 \leq i, j, l \leq K_{k}} \pi_{i j l k} \int_{A_{i k}} \int_{C_{l k}} \int_{B_{j k}} \psi(z)\left(1\left\{\theta_{k}^{(m)}(x, \tau)=2 M_{k}\right\}-\tau\right) \\
& \times \nu_{x}(d x) \nu_{z}(d z) \nu_{y}(d y) \\
= & \sum_{1 \leq i, j, l \leq K_{k}} \pi_{i j l k}\left(\tau \nu_{x}\left\{B_{j k}\right\}-\tau \nu_{x}\left\{B_{j k}\right\}\right) \int_{A_{i k}} \int_{C_{l k}} \psi(z) \nu_{z}(d z) \nu_{y}(d y) \\
= & 0 .
\end{aligned}
$$

In particular, setting $\psi(\cdot)=P_{k}\left\{Y_{i} \leq \theta^{(m)}\left(X_{i}, \tau\right) \mid Z_{i}=\cdot\right\}-\tau$ in (48) implies, by the law of iterated expectations, that $P_{k}\left\{Y_{i} \leq \theta_{k}^{(m)}\left(X_{i}, \tau\right) \mid Z_{i}\right\}=\tau P_{k}$-a.s. for $m \in\{1,2\}$. Note that for each $k, \theta_{k}^{(1)}$ and $\theta_{k}^{(2)}$ can be viewed as functions of $w=\left(x, z^{(1)}\right)$ that only depend on $x$, which together with (47) and (48) imply that $P_{k} \in \mathbf{P}_{0}$ for all $k$. Hence, since $P \in \mathbf{P}_{1}$ was arbitrary and $\left\|P_{k}-P\right\|_{\mathrm{TV}} \rightarrow 0$ as $k \rightarrow \infty$, the conclusion of the theorem follows by Lemma 2.1.

Q.E.D.

PROOF OF THEOREM 3.3: The proof is very similar to that of Theorem 3.2, and we therefore provide only an outline, emphasizing the differences in the arguments. Fixing $P \in \mathbf{P}_{1}$, we may obtain a sequence $\left\{P_{k}\right\}_{k=1}^{\infty}$ such that, for all $k, P_{k} \in \mathbf{M}_{y, x, z}(\nu), d P_{k} / d \nu=f_{k}$ for $f_{k}$ as defined in (43), and such that (42) holds. To show $P_{k} \in \mathbf{P}_{0}$ for all $k$, let $\mathcal{B}\left(B_{j k}\right)$ denote the $\sigma$-algebra generated by all the open subsets of $B_{j k}$. By Assumption 3.3 and Lemma A.1, there then exist $B_{j k}^{(1)}:[0,1] \rightarrow \mathcal{B}\left(B_{j k}\right)$ and $B_{j k}^{(2)}:[0,1] \rightarrow \mathcal{B}\left(B_{j k}\right)$ such that, for all $1 \leq j \leq K_{k}$ and $m \in\{1,2\}$, (i) $\nu_{x}\left\{B_{j k}^{(m)}(\tau)\right\}=\tau \nu_{x}\left\{B_{j k}\right\}$, (ii) $B_{j k}^{(m)}(\tau) \subseteq B_{j k}^{(m)}\left(\tau^{\prime}\right)$ for all $0 \leq \tau \leq$ 
$\tau^{\prime} \leq 1$, and (iii) $\nu_{x}\left\{B_{j k}^{(1)}(\tau) \triangle B_{j k}^{(2)}(\tau)\right\}>0$ for all $\tau \in(0,1)$ and $1 \leq j \leq K_{k}$ such that $\nu_{x}\left\{B_{j k}\right\}>0$. Following (46), we then define functions $\theta_{k}^{(m)}$ pointwise by

$$
\begin{aligned}
\theta_{k}^{(m)}(x, \tau) \equiv & \sum_{j=1}^{K_{k}}\left((2+\tau) M_{k} 1\left\{x \in B_{j k}^{(m)}(\tau)\right\}\right. \\
& \left.-(3-\tau) M_{k} 1\left\{x \in B_{j k} \backslash B_{j k}^{(m)}(\tau)\right\}\right) .
\end{aligned}
$$

Observe that $\left|\theta_{k}^{(m)}(x, \tau)\right| \leq 3 M_{k}$ for all $(x, \tau) \in \mathbf{R}^{d_{x}} \times[0,1]$, and hence $\theta_{k}\left(X_{i}, \tau\right)$ is bounded $P_{k}$-a.s. uniformly in $\tau \in[0,1]$. Moreover, since $B_{j k}^{(m)}(\tau) \subseteq B_{j k}^{(m)}\left(\tau^{\prime}\right)$ for $m \in\{1,2\}$ and all $0 \leq \tau \leq \tau^{\prime} \leq 1$ and $1 \leq j \leq K_{k}$, it follows from (49) and the support of $X_{i}$ under $P_{k}$ being contained in $\left[-M_{k}, M_{k}\right]^{d_{x}}=\bigcup_{j=1}^{K_{k}} B_{j k}$ that $\theta_{k}^{(m)}\left(X_{i}, \tau\right)$ is strictly monotonic in $\tau P_{k}$-a.s. In turn, notice that since $\tau \in[0,1]$ and the support of $Y_{i}$ is contained in $\left[-M_{k}, M_{k}\right]$ under $P_{k}$, we obtain from (49) that, for all $x \in B_{j k}, 1\left\{Y_{i} \leq \theta_{k}^{(m)}(x, \tau)\right\}=1\left\{x \in B_{j k}^{(m)}(\tau)\right\} P_{k}$-a.s. Therefore, arguing as in (47) yields that

$$
E_{P_{k}}\left[\left(1\left\{Y_{i} \leq \theta_{k}^{(1)}\left(X_{i}, \tau\right)\right\}-1\left\{Y_{i} \leq \theta_{k}^{(2)}\left(X_{i}, \tau\right)\right\}\right)^{2}\right]>0,
$$

for all $k$ and $\tau \in(0,1)$. Thus, we may conclude from $(50)$ that $\theta_{k}^{(1)}(\cdot, \tau)$ is distinct from $\theta_{k}^{(2)}(\cdot, \tau)$ under $\|\cdot\|_{L^{\infty}\left(P_{k}\right)}$ for all $\tau \in(0,1)$. In turn, arguing as in (48) further implies that, for all $k$,

$$
P_{k}\left\{Y_{i} \leq \theta_{k}^{(m)}\left(X_{i}, \tau\right) \mid Z_{i}\right\}=\tau
$$

$P_{k}$-a.s. for $m \in\{1,2\}$ and all $\tau \in(0,1)$. Since, for each $k$ and $\tau \in[0,1], \theta_{k}^{(1)}(\cdot, \tau)$ and $\theta_{k}^{(2)}(\cdot, \tau)$ can be viewed as functions of $w=\left(x, z^{(1)}\right)$ that only depend on $x$, results (50) and (51) imply that $P_{k} \in \mathbf{P}_{0}$ for all $k$. The argument can then be finished as in the proof of Theorem 3.2.

Q.E.D.

\section{REFERENCES}

AI, C., AND X. CHEN (2003): "Efficient Estimation of Models With Conditional Moment Restrictions Containing Unknown Functions,” Econometrica, 71, 1795-1843. [2537]

Aliprantis, C. D., AND K. C. Border (2006): Infinite Dimensional Analysis-A Hitchhiker's Guide. Springer, Berlin. [2551]

Anderson, R. M., AND W. R. ZAME (2001): "Genericity With Infinitely Many Parameters," Advances in Theoretical Economics, 1, 1-62. [2544]

ANDERson, T. W. (1951): "Estimating Linear Restrictions on Regression Coefficients for Multivariate Normal Distributions," The Annals of Mathematical Statistics, 22, 327-351. [2544]

(1967): "Confidence Limits for the Expected Value of an Arbitrary Random Variable With a Continuous Distribution Function," Bulletin of the International Statistical Institute, 43, 249-251. [2543] 
ANDERSON, T. W., AND H. RUBin (1949): "Estimation of the Parameters of a Single Equation in a Complete System of Stochastic Equations," The Annals of Mathematical Statistics, 20, 46-63. [2537]

ANDREWS, D. W. K. (2011): "Examples of $L^{2}$-Complete and Boundedly-Complete Distributions," Discussion Paper 1801, Cowles Foundation. [2537,2541,2544,2548]

ANDREWs, D. W. K., AND P. GugGenberger (2009): "Validity of Subsampling and "Plug-in Asymptotic" Inference for Parameters Defined by Moment Inequalities," Econometric Theory, 25, 669-709. [2540]

ANDREws, D. W. K., AND G. SoARes (2010): "Inference for Parameters Defined by Moment Inequalities Using Generalized Moment Selection," Econometrica, 78, 119-157. [2540]

BAHADUR, R. R., AND L. J. SAVAGE (1956): "The Nonexistence of Certain Statistical Procedures in Nonparametric Problems," The Annals of Mathematical Statistics, 27, 1115-1122. [2537,2540]

BERRY, S. T., AND P. A. HAILE (2009): "Identification of a Heterogeneous Generalized Regression Model With Group Effects,” Discussion Paper 1732, Cowles Foundation. [2536]

(2010a): "Identification in Differentiated Products Markets Using Market Level Data," Discussion Paper 1744, Cowles Foundation. [2536]

(2010b): "Nonparametric Identification of Multinomial Choice Demand Models With Heterogeneous Consumers," Discussion Paper 1718, Cowles Foundation. [2536]

Blundell, R., X. ChEN, AND D. KRISTENSEN (2007): "Semi-Nonparametric IV Estimation of Shape-Invariant Engel Curves," Econometrica, 75, 1613-1669. [2535]

BogACHEV, V. I. (2007a): Measure Theory, Vol. I. Berlin: Springer. [2553]

(2007b): Measure Theory, Vol. II. Berlin: Springer. [2549,2551]

CHEN, X., AND D. Pouzo (2012): "Estimation of Nonparametric Conditional Moment Models

With Possibly Nonsmooth Generalized Residuals,” Econometrica, 80, 277-321. [2536]

Chen, X., E. TAMer, AND A. TORgovitsky (2011): "Sensitivity Analysis in Semiparametric Likelihood Models," Discussion Paper 1836, Cowles Foundation. [2537]

CHEN, X., V. CHERNOZHUKOV, S. LEE, AND W. NEWEY (2012): "Local Identification of Nonparametric and Semiparametric Models," Unpublished Manuscript, MIT. [2537,2544,2548]

Chernozhukov, V., AND C. HANSEN (2005): "An IV Model of Quantile Treatment Effects," Econometrica, 73, 245-261. [2536,2546]

Chernozhukov, V., C. HANSEN, AND M. JANSson (2007): "Inference Approaches for Instrumental Variable Quantile Regression," Economics Letters, 95, 272-277. [2537]

(2009): "Finite Sample Inference for Quantile Regression Models," Journal of Econometrics, 152, 93-103. [2537]

Chernozhukov, V., H. Hong, AND E. TAMER (2007): "Estimation and Confidence Regions for Parameter Sets in Econometric Models," Econometrica, 75, 1243-1284. [2537]

CRAGG, J. G., AND S. G. DONALD (1993): "Testing Identifiability and Specification in Instrumental Variable Models," Econometric Theory, 9, 222-240. [2544]

(1997): "Inferring the Rank of a Matrix," Journal of Econometrics, 76, 223-250. [2544]

Cunha, F., J. J. HeCKMAN, AND S. M. SCHENNACH (2010): "Estimating the Technology of Cognitive and Noncognitive Skill Formation," Econometrica, 78, 883-931. [2536]

DAROlles, S., Y. FAN, J. P. FlORENS, AND E. RENAUlt (2011): "Nonparametric Instrumental Regression," Econometrica, 79, 1541-1565. [2536]

D'Haultfoeuille, X. (2011): "On the Completeness Condition in Nonparametric Instrumental Problems," Econometric Theory, 27, 460-471. [2541]

D’HAultFoeuille, X., AND P. FÉvrIER (2012): "Identification of Nonseparable Models With Endogeneity and Discrete Instruments," Unpublished Manuscript, CREST. [2536]

DONOHO, D. L. (1988): "One-Sided Inference About Functionals of a Density," The Annals of Statistics, 16, 1390-1420. [2539]

Dufour, J.-M. (2003): "Identification, Weak Instruments, and Statistical Inference in Econometrics," Canadian Journal of Economics, 36, 767-808. [2537]

FreYberGeR, J., AND J. L. HOROWITZ (2012): "Identification and Shape Restrictions in Nonparametric Instrumental Variables Estimation,” Working Paper CWP15/12, Cemmap. [2537] 
GAGLIARDinI, P., AND O. SCAILLET (2012): "Nonparametric Instrumental Variable Estimation of Structural Quantile Effects," Econometrica, 80, 1533-1562. [2536]

GILL, L., AND A. LEWBEL (1992): "Testing the Rank and Definiteness of Estimated Matrices With Applications to Factor, State-Space and ARMA Models," Journal of the American Statistical Association, 87, 766-776. [2544]

GINÉ, E., AND R. NICKL (2010): “Confidence Bands in Density Estimation,” The Annals of Statistics, 38, 1122-1170. [2540]

HALl, P., AND J. L. HoROwITZ (2005): "Nonparametric Methods for Inference in the Presence of Instrumental Variables," The Annals of Statistics, 33, 2904-2929. [2535,2541]

Hengartner, N. W., AND P. B. STARK (1995): "Finite Sample Confidence Envelopes for ShapreRestricted Densities," The Annals of Statistics, 23, 525-550. [2540]

Hoderlein, S., L. NeSHeIM, AND A. SimONI (2012): "Semiparametric Estimation of Random Coefficients in Structural Economic Models," Working Paper CWP09/12, Cemmap. [2537]

Hoeffding, W., AND J. Wolfowitz (1958): "Distinguishability of Sets of Distributions," The Annals of Mathematical Statistics, 29, 700-718. [2552]

HorowitZ, J. L., AND S. LEE (2007): "Nonparametric Instrumental Variables Estimation of a Quantile Regression Model," Econometrica, 75, 1191-1208. [2536,2541]

HU, Y., AND S. M. SCHENNACH (2008): "Instrumental Variable Treatment of Nonclassical Measurement Error Models," Econometrica, 76, 195-216. [2536]

HunT, B. R., T. SAUER, AND J. A. YoRKE (1992): "Prevalence: A Translation-Invariant "Almost Every" on Infinite-Dimensional Spaces," Bulletin (New Series) of the American Mathematical Society, 27, 217-238. [2544]

IMBENS, G. W., AND W. K. NEWEY (2009): "Identification and Estimation of Triangular Simultaneous Equations Models Without Additivity," Econometrica, 77, 1481-1512. [2536]

KLEIBERGEN, F. (2002): "Pivotal Statistics for Testing Structural Parameters in Instrumental Variables Regression,” Econometrica, 70, 1781-1803. [2537]

Kleibergen, F., AND R. PAAP (2006): "Generalized Reduced Rank Tests Using the Singular Value Decomposition," Journal of Econometrics, 133, 97-126. [2544]

LE CAM, L. (1986): Asymptotic Methods in Statistical Decision Theory. New York: Springer. [2539]

LEEB, H., AND B. M. PÖTSCHER (2008): "Can One Estimate the Unconditional Distribution of Post-Model-Selection Estimators?” Econometric Theory, 24, 338-376. [2537]

LehmanN, E. L., AND H. SChefFÉ (1950): "Completeness, Similar Regions, and Unbiased Estimation-Part I," Sankhya, 10, 305-340. [2535]

(1955): "Completeness, Similar Regions, and Unbiased Estimation—Part II," Sankhya, 15, 219-236. [2535]

MoreIRA, M. J. (2003): "A Conditional Likelihood Ratio Test for Structural Models," Econometrica, 71, 1027-1048. [2537]

MÜLLER, U. K. (2008): "The Impossibility of Consistent Discrimination Between I(0) and I(1) Processes," Econometric Theory, 24, 616-630. [2537]

NEWEY, W. K., AND J. L. POWELL (2003): "Instrumental Variable Estimation of Nonparametric Models," Econometrica, 71, 1565-1578. [2535,2542-2544]

PÖTSCHER, B. M. (2002): "Lower Risk Bounds and Properties of Confidence Sets for Ill-Posed Estimation Problems With Applications to Spectral Density and Persistence Estimation, Unit Roots, and Estimation of Long Memory Parameters," Econometrica, 70, 1035-1065. [2538]

RoBIN, J.-M., AND R. J. SMith (2000): “Tests of Rank,” Econometric Theory, 16, 151-175. [2544]

ROBINS, J., AND A. W. VAN DER VAART (2006): "Adaptive Nonparametric Confidence Sets," The Annals of Statistics, 34, 229-253. [2540]

Romano, J. P. (2004): "On Non-Parametric Testing, the Uniform Behavior of the $t$-Test, and Related Problems," Scandinavian Journal of Statistics, 31, 567-584. [2537,2539,2540,2552]

ROMANO, J. P., AND A. M. SHAIKH (2008): "Inference for Identifiable Parameters in Partially Identified Econometric Models," Journal of Statistical Planning and Inference, 138, 2786-2807. $[2537,2540]$ 
(2012): "On the Uniform Asymptotic Validity of Subsampling and the Bootstrap," The Annals of Statistics, 40, 2798-2822. [2540]

Romano, J. P., AND M. WOLF (2000): "Finite Sample Nonparametric Inference and Large Sample Efficiency," The Annals of Statistics, 28, 756-778. [2543]

SANTOS, A. (2012): "Inference in Nonparametric Instrumental Variables With Partial Identification," Econometrica, 80, 213-275. [2537,2543]

TORGOVITSKY, A. (2012): "Identification of Nonseparable Models With General Instruments," Working Paper, Northwestern University. [2536]

Dept. of Economics, Northwestern University, 2001 Sheridan Road, Evanston, IL 60208, U.S.A.; iacanay@northwestern.edu,

Dept. of Economics, University of California-San Diego, 9500 Gilman Drive \#0508, La Jolla, CA 92093, U.S.A.; a2santos@ucsd.edu,

and

Dept. of Economics, University of Chicago, 1126 E. 58th Street, Chicago, IL 60657,U.S.A.; amshaikh@uchicago.edu.

Manuscript received June, 2012; final revision received April, 2013. 